\title{
65. A Case of a Female Infant with Terminal Deletion of the Long Arm of No. 4 Chromosome
}

\author{
By Tetsuji Kadotani, ${ }^{* 1}$ Yoko Watanabe,*) Shigeki TaKeUChI, ${ }^{* *}$ \\ Michiko HaYashidani, ${ }^{* *}$ and Satoru OHYA***) \\ (Communicated by Sajiro Makıno, M. J. A., Sept. 12, 1986)
}

Up to date, the deletions of the long arm of no. 4 chromosome were reported on 23 cases by the several authors (Ockey et al. 1967; Golbus et al. 1973; Ferrier and Freund 1974; v. Kempen 1975; Back et al. 1977; Serville and Broustet 1977; Frias et al. 1978; Rethoré et al. 1979; Townes et al. 1979; Davis et al. 1981; Fryns et al. 1981; Mitchell et al. 1981; Yu et al. 1981; Lech et al. 1982; Lipson et al. 1982; Stamberg et al. 1982). Of these, 5 cases had the interstitial deletions of $4 \mathrm{q}$, and 18 cases had the terminal deletions of $4 \mathrm{q}$. In this paper, we reported on an additional female infant with the terminal deletion at $4 \mathrm{q} 31$.

Case report. The propositus was a female infant of 15 days after delivery, and she was born as the first child between a 25 -year-old mother and 34-year-old father. There was no history of abortion, still birth, congenital malformations, consanguinity, and the atomic bomb exposure in this couple. The clinical examination revealed her both parents were phenotypically normal showing no evidence for the mental deficiency.

The birth weight of the propositus was $2900 \mathrm{~g}$ at 38 weeks of the gestation. Her remarkable clinical findings were short neck, hypertelorism, epicanthus, low set ears, low nasal bridge, saddle nose, cleft lip and cleft palate, hypoplastic mandible, low voice, wide spaced nipples, clinodactyly of the 4th and 5th fingers of her right hand, peculiar overlapping at her right hand, poorly developed flexion creases in the 5th finger of her right hand, low set in the 5th fingers, deep D-lines of both palms, flat feet, abnormal positions of toes, hypotonia, sinking breathing due to the upper airway stenosis (Figs. 1, 2).

Cytological findings. Chromosome slides for this study were prepared from the leucocyte cultures established from the propositus and her parents. The G-banding differential staining was applied for the chromosome identification. The chromosome numbers were determined with 124 well-delineated metaphases. Karyotypes were analysed in 12 cells each following the conventional Giemsa and the G-banding procedures.

The karyotype of the propositus based on the conventional Giemsa specimen showed 46 chromosomes including a no. 4 chromosome having an unusually shortened long arm. By the analysis of G-banding methods, it was revealed that an unusual element corresponded to the segment $q 31 \rightarrow$ qter of no. 4 chromosome. Then the chromosome formula of the propositus was given as 46,XX,del (4) (q31) (Fig. 3). Both parents of the propositus were chromosomally normal with no slight evidence for the aberration, based on 12 cells karyotyped in each case.

*) The Kadotani Medical Research Foundation, 1248, Saijohigashi, Saijo, Higashihiroshima 724, Japan.

**) Department of Neonatology, Hiroshima City Hospital, Hiroshima, Japan.

***) Ohya Clinic, Kuchida 4-20-13, Asakita-ku, Hiroshima, Japan. 


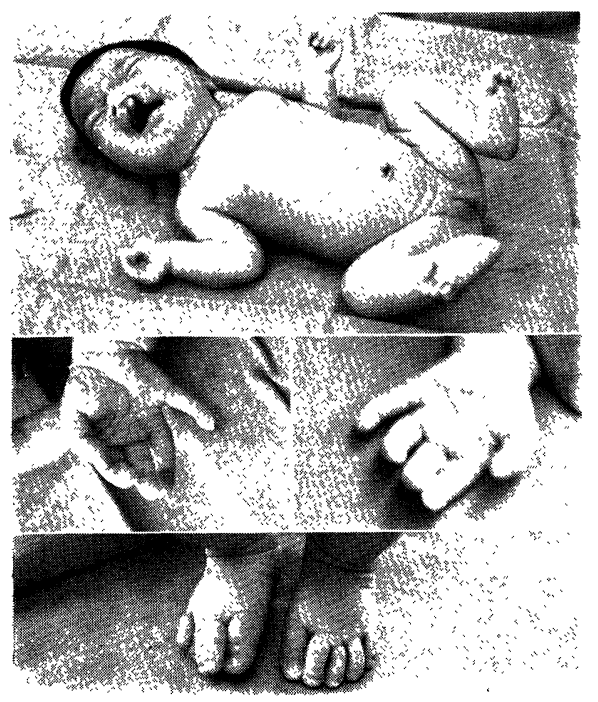

Fig. 1

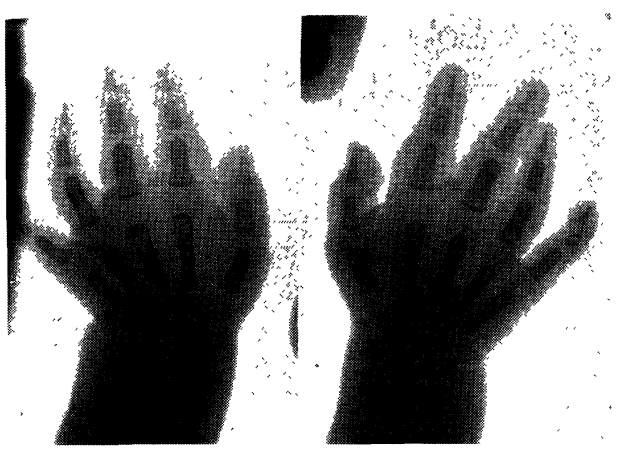

Fig. 2

Figs. 1-2. 1: Clinical features of the propositus. 2: X-ray finding of the propositus' hands.

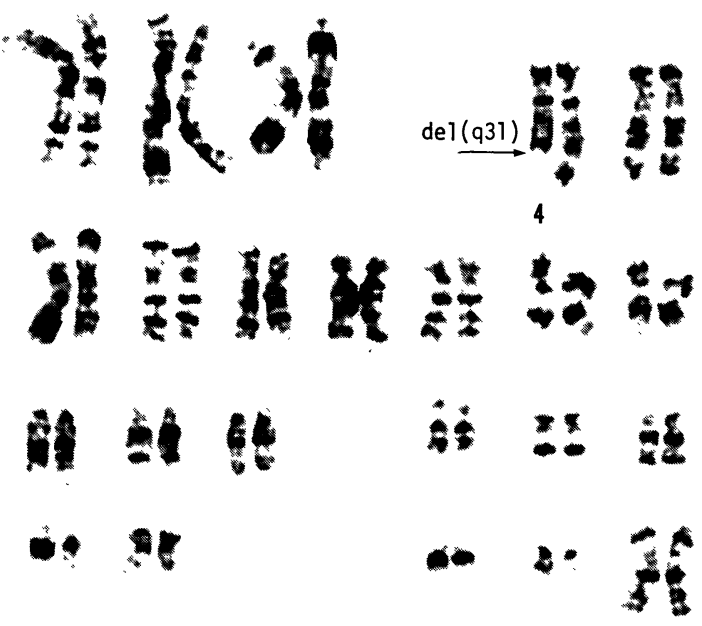

Fig. 3. G-banding karyotype of the propositus (46,XX,del(4) (q31)).

Remarks and conclusion. The first initial case of del(4q) was reported by Ockey et al. (1967). Thereafter additional cases for the deletion of the long arm of no. 4 chromosome using the G-bands were reported by several authors (Golbus et al. 1973; Ferrier and Freund 1974; v. Kempen 1975; Back et al. 1977; Serville and Broustet 1977; Frias et al. 1978; Rethoré et al. 1979; Townes et al. 1979; Davis et al. 1981; Fryns et al. 1981; Mitchell et al. 1981; Yu et al. 1981; Lech et al. 1982; Lipson et al. 1982; Stamberg et al. 1982).

The deleted portions of the long arm of no. 4 chromosome were divided into two groups which were the interstitial deletion and the terminal deletion 
Table I. The case of deletion of the long arm of no. 4 chromosome

\begin{tabular}{|c|c|c|}
\hline $\begin{array}{l}\text { Part of } \\
\text { deletion }\end{array}$ & $\begin{array}{l}\text { No. of } \\
\text { cases }\end{array}$ & Authors \\
\hline Interstitial deletion & & \\
\hline $\mathrm{q} 12 \rightarrow \mathrm{q} 21$ & 1 & Lech et al. (1982) \\
\hline $\mathrm{q} 21 \rightarrow \mathrm{q} 25$ & 1 & Mitchell et al. (1981) \\
\hline $\mathrm{q} 21 \rightarrow \mathrm{q} 26$ & 1 & Mitchell et al. (1981) \\
\hline $\mathrm{q} 24 \rightarrow \mathrm{q} 32$ & 1 & Serville \& Broustet (1977) \\
\hline $\mathrm{q} 27 \rightarrow \mathrm{q} 31$ & 1 & Mitchell et al. (1981) \\
\hline $\begin{array}{l}\text { Terminal deletion: } \\
\text { q3 or } \mathrm{q} 31 \rightarrow \mathrm{qter}\end{array}$ & 15 & $\begin{array}{l}\text { Ockey et al. (1967), Golbus et al. (1973), } \\
\text { Ferrier et al. (1974), v. Kempen (1975), } \\
\text { Back et al. (1977), Frias (1978), } \\
\text { Townes et al. (1979), Yu et al. (1981), } \\
\text { Davis et al. (1981), Mitchell et al. (1981), } \\
\text { Lipton et al. (1982), Present case }\end{array}$ \\
\hline $\mathrm{q} 32 \rightarrow$ qter & 2 & Rethoré et al. (1979), Fryns et al. (1981) \\
\hline q33 $\rightarrow$ qter & 2 & Mitchell et al. (1981), Stamberg et al. (1982) \\
\hline
\end{tabular}

(Table I). On the interstitial deletion, it was reported on 5 cases: One case with the deleted segment of $\mathrm{q} 12 \rightarrow \mathrm{q} 21$ (Lech et al. 1982), one case of $\mathrm{q} 21 \rightarrow \mathrm{q} 25$ (Mitchell et al. 1981), one case of $q 21 \rightarrow q 26$ (Mitchell et al. 1981), one case of $\mathrm{q} 24 \rightarrow \mathrm{q} 32$ (Serville and Broustet, 1977), and one case of $\mathrm{q} 27 \rightarrow \mathrm{q} 31$ (Mitchell et al. 1981). On the terminal deletion, it was reported on 19 cases: 15 cases with the deleted segment of $\mathrm{q} 3 \rightarrow$ qter or $\mathrm{q} 31 \rightarrow \mathrm{qter}$ (Ockey et al. 1967; Golbus et al. 1973; Ferrier et al. 1974; v. Kempen 1975; Back et al. 1977; Frias et al. 1978; Townes et al. 1979; Yu et al. 1981; Davis et al. 1981; Mitchell et al. 1981; Lipson et al. 1982 ; the present case), two cases of q32 $\rightarrow$ qter (Rethoré et al. 1979 ; Fryns et al. 1981), and two cases of q33 $\rightarrow$ qter (Mitchell et al. 1981; Stamberg et al. 1982).

The deleted segment of $4 \mathrm{q} 3 \rightarrow \mathrm{qter}$ and the manifestations of all those reported cases are summarized in Table II. Male cases were seven of them and female cases were twelve. All the patients were born at full term or near full term with the normal birth weight except for one case by Townes et al. (1979) in which the deletion resulted from a paternal translocation.

According to Table II, the characteristic features of $4 q$-syndrome were retrognathia or micrognathia $(89 \%)$, cleft lip and/or cleft palate $(79 \%)$, flat nasal bridge $(79 \%)$, short nose $(74 \%)$, low set and/or malformed ears $(74 \%)$, displacement of toes $(68 \%)$, clinodactyly of fingers and/or toes $(58 \%)$, hypertelorism $(58 \%)$, epicanthus $(53 \%)$, cardiac anomalies $(53 \%)$ and the growth retardation $(53 \%)$. In parental karyotypes, 17 cases out of 19 cases with the terminal deletion were normal. One case reported by Townes et al. (1979) had the abnormal karyotype and resulted from a paternal translocation between no. 4 and no. 20 chromosomes. And on one case reported by Fryns et al. (1981), the chromosomal examination of her parents was not cooperated.

Acknowledgements. We are cordially obliged to Emeritus Professor Sajiro Makino, M. J. A., the senior director of the Kadotani Medical Research Foundation, for improvement of this manuscript. Financial aid from the Japan Academy is gratefully acknowledged here. 
Table II. Manifestations of the deletion of the long arm of no. 4 chromosome

\begin{tabular}{|c|c|c|c|c|c|c|c|c|c|c|c|}
\hline Symptoms and findings & $\begin{array}{l}\text { Ockey } \\
\text { ct al. } \\
(1967)\end{array}$ & \multicolumn{2}{|c|}{$\begin{array}{l}\text { Golbus } \\
\text { ct al. } \\
(1973) \\
\end{array}$} & $\begin{array}{c}\text { Ferrier \& } \\
\text { Frreund } \\
(1974)\end{array}$ & $\begin{array}{l}\text { v. Kempen } \\
(1975) \\
\end{array}$ & $\begin{array}{l}\mathrm{Ba} \\
\text { et } \\
(19\end{array}$ & $\begin{array}{l}\text { Back } \\
\text { et al. } \\
\text { (1977) }\end{array}$ & $\begin{array}{l}\text { Frias } \\
\text { et al. } \\
(1978)\end{array}$ & $\begin{array}{l}\text { Townes } \\
\text { ct al. } \\
\text { (1979) }\end{array}$ & $\begin{array}{l}\mathrm{Yu} \\
\text { et } a l . \\
(1981)\end{array}$ & $\begin{array}{l}\text { Davis } \\
\text { et al. } \\
(1981)\end{array}$ \\
\hline Deletion & $4 q$ & $4 \mathrm{q}$ & & $4 q 3$ & $4 \mathrm{q} 31$ & & $q 31$ & $4 \mathrm{q} 31$ & $4 q 31$ & $4 \mathrm{q} 31$ & $4 \mathrm{q} 31$ \\
\hline Sex & $\mathrm{M}$ & $\mathrm{F}$ & & $F$ & $\mathrm{M}$ & & $\mathrm{F}$ & $\mathrm{M}$ & $\mathrm{M}$ & M & $\mathrm{F}$ \\
\hline Gestation (weeks) & 40 & 40 & & 40 & 36 & & 40 & 38 & 30 & 37 & 40 \\
\hline Birth weight $(g)$ & 3600 & 336 & & 3120 & 2430 & & 2900 & 2600 & 1295 & 2400 & 3530 \\
\hline Age at death & $3.5 \mathrm{~m}$. & & & $4 \mathrm{~m}$. & $(6 \mathrm{y})$. & & 5 y.) & $6 \mathrm{~m}$. & $1 \mathrm{hr}$. & $17 \mathrm{~d}$. & $(19 \mathrm{~m})$. \\
\hline Growth retardation & & & & + & + & & - & + & & + & + \\
\hline Mental retardation & & \pm & & \pm & + & & + & + & & & + \\
\hline Abnormal/asymmetric head & & & & + & + & & + & + & + & + & \\
\hline Microcephaly & & & & - & + & & + & & & & - \\
\hline Short neck & & & & & & & & & & + & \\
\hline $\begin{array}{l}\text { Upward slanted palpebral } \\
\text { fissure }\end{array}$ & + & & & + & - & & - & + & & + & + \\
\hline Hypertelorism & + & & & & + & & + & & + & + & + \\
\hline Epicanthus & & & & + & + & & + & + & & + & \\
\hline $\begin{array}{l}\text { Low set and/or malformed } \\
\text { ears }\end{array}$ & + & & & + & - & & - & + & + & + & + \\
\hline Soarting ears & & + & & & + & & - & + & + & - & \\
\hline Flat nasal bridge & + & & & + & + & & + & + & & + & + \\
\hline Short nose & + & + & & + & - & & - & + & + & + & + \\
\hline Cleft lip and'or cleft palate & & + & & + & + & & + & + & + & + & + \\
\hline Retro-/Micrognathia & & + & & + & + & & - & + & + & + & + \\
\hline Facial bone hypoplasia & & + & & + & + & & & + & + & & \\
\hline $\begin{array}{l}\text { Poorly developed flexion } \\
\text { creases in the } 5 \text { th fingers }\end{array}$ & & + & & + & + & & - & + & + & - & + \\
\hline Simian creases & & & & + & + & & + & + & + & + & - \\
\hline Clinodactyly (fingers, toes) & & & & + & + & & & + & + & - & + \\
\hline Syndactyly (fingers, toes) & + & & & & + & & + & + & & & \\
\hline Displacement of toes & + & + & & + & - & & - & & + & & + \\
\hline Cardiac anomalies & + & + & & + & + & & - & & & + & + \\
\hline Renal anomalies & + & + & & + & - & & & & & - & - \\
\hline Parental karyotype & Normal & Norr & mal & Normal & Normal & & rmal & Normal & $\mathrm{t}(4 ; 20)$ pat & Normal & Normal \\
\hline Symptoms and findings & & itchell $c t$ & al. $(198$ & 981) & $\begin{array}{l}\text { Lipson } \mathrm{P} \\
\text { et al. } \\
\text { (1982) }\end{array}$ & $\begin{array}{c}\text { Present } \\
\text { case } \\
(1986) \\
\end{array}$ & $\begin{array}{c}\text { Rethoré } \\
\text { et al. } \\
\text { (1979) } \\
\end{array}$ & $\begin{array}{l}\text { Fryns } \\
\text { et al. } \\
\text { (1981) }\end{array}$ & $\begin{array}{c}\text { Mitchell } \\
\text { et al. } \\
\text { (19) } 81 \\
\end{array}$ & $\begin{array}{l}\text { Stamberg } \\
\text { et al. } \\
(1982) \\
\end{array}$ & Total $(\%)$ \\
\hline Deletion & $4 \mathrm{q} 31.3$ & $4 \mathrm{q} 31.5$ & $4 \mathrm{q} 31.5$ & $4 q 31.5$ & $4 q 31$ & $4 \mathrm{q} 31$ & $4 \mathrm{q} 32.1$ & $4 \mathrm{q} 32$ & $4 \mathrm{q} 33$ & $4 \mathrm{q} 33$ & \\
\hline Sex & $\mathrm{F}$ & $\mathrm{F}$ & $\mathrm{F}$ & $\mathrm{F}$ & $F$ & $\mathbf{F}$ & $\mathrm{M}$ & $\mathrm{F}$ & $F$ & M & \\
\hline Gestation (weeks) & 36 & 40 & 40 & 39 & 40 & 38 & 37 & 38 & 40 & 32 & \\
\hline Birth weight (g) & 2300 & 3200 & 2720 & 2600 & 3100 & 2900 & 2800 & 2000 & 3100 & 2000 & \\
\hline Age at death & $1 \mathrm{hr}$. & $(21 \mathrm{~m})$. & $15 \mathrm{~m}$ & $23 \mathrm{~d}$. & $(9 \mathrm{y})$. & $(15 \mathrm{~d})$. & $3 \mathrm{~m}$. & $(12 \mathrm{y.})$ & $(12 \mathrm{y})$. & $(11 \mathrm{~m})$. & \\
\hline Growth retardation & + & + & & + & & & & & + & + & $10 / 19(53)$ \\
\hline Mental retardation & + & + & & + & & & & + & \pm & & $\begin{array}{l}11 \sim 8 / 19 \\
(58 \sim 42)\end{array}$ \\
\hline Abnormal/asymmetric head & & & & & & & & & - & + & $7 / 19(37)$ \\
\hline Microcephaly & & + & & + & & & & & - & + & $5 / 19(26)$ \\
\hline Short neck & + & - & - & + & & + & + & - & - & & $5 / 19(26)$ \\
\hline $\begin{array}{l}\text { Upward slanted palpebral } \\
\text { fissure }\end{array}$ & - & + & - & - & & & & & + & & $7 / 19(37)$ \\
\hline Hypertelorism & + & + & - & - & & + & + & & - & + & $11 / 19(58)$ \\
\hline Epicanthus & - & + & + & - & & + & + & & + & - & $10 / 19(53)$ \\
\hline $\begin{array}{l}\text { Low set and/or malformed } \\
\text { ears }\end{array}$ & + & + & + & + & + & + & + & + & - & - & $14 / 19(74)$ \\
\hline Soarting ears & & & & & + & & & & - & & $5 / 19(26)$ \\
\hline Flat nasal bridge & + & + & + & + & + & + & & & + & + & $15 / 19(79)$ \\
\hline Short nose & + & + & + & + & & & + & + & + & & $14 / 19(74)$ \\
\hline Cleft lip and/or cleft palate & + & + & - & + & + & + & + & + & - & - & $15 / 19(79)$ \\
\hline Retro-/Micrognathia & + & + & + & + & + & + & + & + & + & + & $17 / 19(89)$ \\
\hline Facial bone hypoplasia & - & - & + & + & + & & & & - & & $8 / 19(42)$ \\
\hline $\begin{array}{l}\text { Poorly developed flexion } \\
\text { creases in the } 5 \text { th fingers }\end{array}$ & & & & & & + & + & & - & + & $9 / 19(47)$ \\
\hline Simian creases & + & + & + & - & & - & & & - & & $9 / 19(47)$ \\
\hline Clinodactyly (fingers, toes) & & + & + & & + & + & + & & - & + & $11 / 19(58)$ \\
\hline Syndactyly (fingers, toes) & & & & & - & - & & & - & + & $5 / 19(26)$ \\
\hline Displacement of toes & + & + & + & + & & + & + & & + & + & $13 / 19(68)$ \\
\hline Cardiac anomalies & - & + & + & + & - & - & + & - & - & - & $10 / 19(53)$ \\
\hline Renal anomalies & - & + & - & - & & & & & - & & $4 / 19(21)$ \\
\hline
\end{tabular}

( ) : Alive. y., years; m., months; d., days; hr., hours. 


\section{References}

Back, E. et al. (1977) : Ann. Génét., 20, 294-296.

Davis, J. M. et al. (1981) : Amer. J. Med. Genet., 9, 113-117.

Ferrier, S., and Freund, M. (1974) : Arch. Genet., 47, 16-26.

Frias, J. L. et al. (1978) : Birth Defects, XIV, 355-358.

Fryns, J. P. et al. (1981) : Ann. Génét., 24, 187-188.

Golbus, M. S. et al. (1973) : J. Med. Genet., 10, 83-85.

Lech, H. et al. (1982) : Klin. Pädiat., 194, 117-119.

Lipson, A. et al. (1982) : J. Med. Genet., 19, 155-157.

Mitchell, L. A. et al. (1981): Amer. J. Med. Genet., 8, 73-89.

Ockey, C. H. et al. (1967) : Arch. Dis. Child., 42, 428-434.

Rethoré, M. O. et al. (1979) : Ann. Génét., 22, 214-216.

Serville, F., and Broustet, A. (1977) : Hum. Genet., 39, 239-242.

Stamberg, J. et al. (1982) : Clin. Genet., 21, 125-129.

Townes, P. L. et al. (1979) : Am. J. Dis. Child., 133, 383-385.

van Kempen, C. (1975): J. Med. Genet., 12, 204-207.

Yu, C. W. et al. (1981): Ann. Génét., 24, 158-161. 